\title{
SELF-SIMILARITY IN VISCOUS BOUSSINESQ EQUATIONS
}

\author{
GRZEGORZ KARCH AND NICOLAS PRIOUX
}

(Communicated by David S. Tartakoff)

\begin{abstract}
We study the existence and the asymptotic stability as the time variable escapes to infinity of self-similar solutions to the viscous Boussinesq equations posed in the whole three dimensional space.
\end{abstract}

\section{INTRODUCTION}

In the natural or free convective transfer, the heat is transported between a solid surface and a fluid moving over it. Here, the fluid motion is entirely caused by the buoyancy forces arising from density changes that result from the temperature variations in the fluid. The fluid movement by the natural convection can be either laminar or turbulent. However, because of low velocities that usually exist in the natural convection, laminar flow occurs more frequently. For these reasons, we will focus on laminar natural convective flow. The reader is referred to the classical book $[8, \mathrm{ch} .5, \S 56]$ as well as to $[1, \mathrm{ch} .4 .2]$ for more details on models describing the heat transport in a fluid. Following [8] and putting all physical constants equal to 1 , the initial value problem for the viscous Boussinesq equations, modeling the phenomena presented above, has the form

$$
\begin{aligned}
& v_{t}+(v \cdot \nabla) v+\nabla p=\Delta v-\beta T+F, \\
& \nabla \cdot v=0, \\
& T_{t}+v \cdot \nabla T=\Delta T, \\
& v(0, x)=v_{0}(x), \quad T(0, x)=T_{0}(x) .
\end{aligned}
$$

Here, the time variable $t$ belongs to the interval $] 0, \infty[$, the space variable $x$ lives in the whole space $\mathbb{R}^{3}$, the vector $v=\left(v_{1}(t, x), v_{2}(t, x), v_{3}(t, x)\right)$ is the unknown velocity of the fluid, and the scalar function $T=T(t, x)$ is the unknown temperature. Moreover, $F=F(t, x)$ is a given external force, the constant vector $\beta \in \mathbb{R}^{3}$ is proportional to the thermal expansion coefficient of the fluid and to the gravitational force. Finally, the functions $v_{0}$ and $T_{0}$ denote given initial conditions.

Mathematical results on the existence and the uniqueness of solutions to system (1.1) can be found in $[2,6]$ and the references therein. Here we note that (1.1) is

Received by the editors July 5, 2006.

2000 Mathematics Subject Classification. Primary 35Q30; Secondary 35B40, 76D05.

Key words and phrases. Natural convection, Boussinesq system, self-similar solutions, large time asymptotics.

The preparation of this paper by the first author was partially supported by the European Commission Marie Curie Host Fellowship for the Transfer of Knowledge "Harmonic Analysis, Nonlinear Analysis and Probability" MTKD-CT-2004-013389.

(c) 2007 American Mathematical Society Reverts to public domain 28 years from publication 
invariant under the rescaling

$$
v_{\lambda}(t, x)=\lambda v\left(\lambda^{2} t, \lambda x\right) \quad \text { and } \quad T_{\lambda}(t, x)=\lambda^{3} T\left(\lambda^{2} t, \lambda x\right)
$$

for all $\lambda>0$. Hence, the main goal of this paper is to construct self-similar solutions to system (1.1), that is, solutions satisfying the scaling relation

$$
v(t, x)=\lambda v\left(\lambda^{2} t, \lambda x\right) \quad \text { and } \quad T(t, x)=\lambda^{3} T\left(\lambda^{2} t, \lambda x\right)
$$

for all $\lambda>0, x \in \mathbb{R}^{3}$, and $t>0$ as well as to study their role in the large time behavior of general solutions. We use the tools introduced in [4] where these questions were answered in the case of the Navier-Stokes equations posed in the whole space $\mathbb{R}^{3}$ and supplemented with small initial data belonging to the space $\mathcal{P M}^{2}$; cf. (1.6) below.

We begin our analysis by recalling the Leray projector $\mathbb{P}$ given formally by the formula $\mathbb{P} v=v-\nabla \Delta^{-1}(\nabla \cdot v)$. Hence, this is the pseudodifferential operator with the matrix symbol $(\widehat{\mathbb{P}}(\xi))_{j, k}=\delta_{j k}-\xi_{j} \xi_{k}|\xi|^{-2}$ and we refer the reader to [3] for more details and additional references. Using well-known properties of the projection $\mathbb{P}$ and the equalities $(v \cdot \nabla) v=\nabla \cdot(v \otimes v)$ and $v \cdot \nabla T=\nabla \cdot(v T)$ (being the immediate consequence of the equation $\nabla \cdot v=0$ ), we rewrite system (1.1) as

$$
v_{t}-\Delta v+\mathbb{P} \nabla \cdot(v \otimes v)=-\beta \mathbb{P} T+\mathbb{P} F, \quad T_{t}-\Delta T+\nabla \cdot(v T)=0 .
$$

Moreover, by the Duhamel principle, we obtain the (formally equivalent to (1.1)) system of integral equations

$$
\begin{aligned}
v(t)= & S(t) v_{0}+\int_{0}^{t} S(t-\tau) \mathbb{P} F(\tau) d \tau-\int_{0}^{t} \nabla S(t-\tau) \mathbb{P}(v \otimes v)(\tau) d \tau \\
& -\beta \int_{0}^{t} S(t-\tau) \mathbb{P} T(\tau) d \tau \\
T(t)= & S(t) T_{0}-\int_{0}^{t} \nabla S(t-\tau)(v T)(\tau) d \tau .
\end{aligned}
$$

Here, $S(t)$ denotes the heat semigroup given as the convolution with the GaussWeierstrass kernel. In this paper, we study properties of solutions to the system of equations (1.4)-(1.5) in the spaces defined as follows. For $a \geq 0$, we put

$$
\mathcal{P} \mathcal{M}^{a} \equiv\left\{f \in \mathcal{S}^{\prime}\left(\mathbb{R}^{3}\right), \quad \widehat{f} \in L_{\text {loc }}^{1}\left(\mathbb{R}^{3}\right), \quad\|f\|_{\mathcal{P M}^{a}} \equiv \underset{\xi \in \mathbb{R}^{3}}{\operatorname{ess} \sup }|\xi|^{a}|\widehat{f}(\xi)|<\infty\right\} .
$$

We construct solutions to system (1.4)-(1.5) (or system (1.1)) with the velocity $v$ in the space

$$
\mathcal{X}=\mathcal{C}_{w}\left(\left[0, \infty\left[, \mathcal{P M}^{2}\right)\right.\right.
$$

supplemented with the norm $\|v\|_{\mathcal{X}}=\sup _{\tau>0}\|v(\tau)\|_{\mathcal{P M}^{2}}$ and the temperature $T$ belonging to

$$
\mathcal{Y}_{a}=\left\{T \in \mathcal { C } _ { w } \left(\left[0, \infty\left[, \mathcal{P M}^{0}\right), \quad \sup _{\tau>0} \tau^{a / 2}\|T(\tau)\|_{\mathcal{P M}^{a}}<\infty\right\}\right.\right.
$$

with the norm $\|T\|_{\mathcal{Y}_{a}}=\sup _{\tau>0}\|T(\tau)\|_{\mathcal{P M}^{0}}+\sup _{\tau>0} \tau^{a / 2}\|T(\tau)\|_{\mathcal{P M}^{a}}$.

We recall that $\mathcal{C}_{w}$ denotes the space of vector-valued functions which are weakly continuous in $t$ (cf. [3]). In order to simplify notation, we denote the product of Banach spaces $\mathcal{Z} \times \mathcal{Z} \times \mathcal{Z}$ with the usual norm $\left\|\left(v_{1}, v_{2}, v_{3}\right)\right\|_{\mathcal{Z} \times \mathcal{Z} \times \mathcal{Z}}=$ $\max \left\{\left\|v_{1}\right\|_{\mathcal{Z}},\left\|v_{2}\right\|_{\mathcal{Z}},\left\|v_{3}\right\|_{\mathcal{Z}}\right\}$ also by $\mathcal{Z}$. 
Our main result on the existence of solutions to system (1.1) reads as follows.

Theorem 1.1. Assume that $v_{0} \in \mathcal{P} \mathcal{M}^{2}, T_{0} \in \mathcal{P} \mathcal{M}^{0}$ and $F \in \mathcal{C}_{w}\left(\left[0, \infty\left[, \mathcal{P M}^{0}\right)\right.\right.$. Fix $1<a<2$. Suppose that $|\beta|=\max \left\{\left|\beta_{1}\right|,\left|\beta_{2}\right|,\left|\beta_{3}\right|\right\}<1$. There is $\gamma>0$ such that if $\left\|v_{0}\right\|_{\mathcal{P M}^{2}}+\left\|T_{0}\right\|_{\mathcal{P M}^{0}}+\sup _{\tau>0}\|F(\tau)\|_{\mathcal{P M}^{0}}<\gamma$, then there exists a solution of system (1.4)-(1.5) in the space $\mathcal{X} \times \mathcal{Y}_{a}$ corresponding to the initial datum $\left(v_{0}, T_{0}\right)$. Moreover, there exists another constant $\Gamma>0$ such that this is the unique solution satisfying the condition $\|v\|_{\mathcal{X}}+\|T\|_{\mathcal{Y}_{a}}<\Gamma$.

Remark 1.2. As we shall see below, $\gamma=\alpha_{1}(1-|\beta|)^{2}\left(2 \alpha_{1}+\alpha_{2}\right)^{-2}$ and $\Gamma=$ $(1-|\beta|)\left(2 \alpha_{1}+\alpha_{2}\right)^{-1}$ where the constants $\alpha_{1}$ and $\alpha_{2}$ are obtained in Lemmata 2.3 and 2.4 , respectively.

Now the standard procedure, based on the uniqueness of solutions from Theorem 1.1, allows us to deduce the existence of self-similar solutions to system (1.1).

Corollary 1.3. Assume that $v_{0}, T_{0}$ and $F$ satisfy the assumptions of Theorem 1.1. Let, moreover, $v_{0}$ be homogeneous of degree -1 , let $T_{0}$ be homogeneous of degree -3 , and let $F$ satisfy the relation $\lambda^{3} F\left(\lambda^{2} t, \lambda x\right)=F(t, x)$ for all $\lambda>0, x \in \mathbb{R}^{3}$, and $t>0$. Then, the solution $(v, T)$ constructed in Theorem 1.1 is self-similar, i.e., invariant under the rescaling (1.2).

Remark 1.4. Note that the Dirac delta $\delta_{0} \in \mathcal{P} \mathcal{M}^{0}\left(\mathbb{R}^{3}\right)$ is a homogeneous distribution of degree -3 .

In our next theorem, we study the asymptotic stability of solutions in the sense proposed in [7] and developed in [4, 5].

Theorem 1.5. Assume that $v_{0}, T_{0}, F$ and $\bar{v}_{0}, \bar{T}_{0}, \bar{F}$ satisfy the assumptions of Theorem 1.1 and denote by $(v, T)$ and $(\bar{v}, \bar{T})$ the corresponding solutions of (1.4)(1.5). Suppose, moreover, that

$$
\begin{gathered}
\lim _{t \rightarrow+\infty}\left(\left\|S(t)\left(v_{0}-\bar{v}_{0}\right)\right\|_{\mathcal{P M}^{2}}+\left\|S(t)\left(T_{0}-\bar{T}_{0}\right)\right\|_{\mathcal{P} \mathcal{M}^{0}}\right. \\
\left.+\|F(t)-\bar{F}(t)\|_{\mathcal{P M}^{0}}\right)=0 .
\end{gathered}
$$

Then

$$
\lim _{t \rightarrow \infty}\left(\|v(t)-\bar{v}(t)\|_{\mathcal{P M}^{2}}+\|T(t)-\bar{T}(t)\|_{\mathcal{P M}^{0}}+t^{a / 2}\|T(t)-\bar{T}(t)\|_{\mathcal{P M}^{a}}\right)=0 .
$$

Remark 1.6. The $\mathcal{P} \mathcal{M}^{a}$ spaces seem to be exotic in the study of the nonlinear problem (1.1). However, it was shown in [5] that they appear in a very natural way in estimates of nonlinear terms like those in (1.4) and (1.5). Note, for example, that $L^{1}\left(\mathbb{R}^{3}\right)$ (or more generally any finite measure on $\mathbb{R}^{3}$ ) is contained in $\mathcal{P M}^{0}$. Moreover, repeating the interpolation argument from [5, Lemma 7.4], one can prove that for each $a \in(3 / 2,2), \mathcal{P} \mathcal{M}^{0} \cap \mathcal{P} \mathcal{M}^{a} \subset L^{p}\left(\mathbb{R}^{3}\right)$ for any $p \in[2,3 /(3-a))$, which implies the corresponding regularity result for the temperature $T=T(t, x)$. Finally, by the results from [5, Section 7], we immediately obtain that $\sup _{t>0} t^{(1-3 / q) / 2}\|v(t)\|_{L^{q}\left(\mathbb{R}^{3}\right)}<\infty$ for $q>3$.

Remark 1.7. We refer the reader to [7, Lemma 6.2] and [4, Remark following Theorem 5.1] for a discussion on the assumption (1.7). In particular, one can easily show that $\left\|S(t)\left(M \delta_{0}-\bar{T}_{0}\right)\right\|_{\mathcal{P M}^{0}} \rightarrow 0$ as $t \rightarrow \infty$ for any $\bar{T}_{0} \in L^{1}\left(\mathbb{R}^{3}\right)$ such that $\int_{\mathbb{R}^{3}} \bar{T}_{0}(x) d x=M$. 


\section{The EXISTENCE OF SOLUTiOns}

The system of integral equations (1.4)-(1.5) can be written in the abstract form

$$
v=y_{1}+B_{1}(v, v)+L T, \quad T=y_{2}+B_{2}(v, T),
$$

where $y_{1}=S(t) v_{0}+\int_{0}^{t} S(t-\tau) \mathbb{P} F(\tau) d \tau, y_{2}=S(t) T_{0}, B_{1}(v, v)$ and $L T$ are the third and fourth terms on the right-hand side of equation (1.4), and $B_{2}(v, T)$ is the second term on the right-hand side of equation (1.5). Solutions to the abstract system (2.1) can be constructed via the Banach fixed point algorithm and such problems were studied in [9]. Hence, we shall only sketch such a reasoning and we refer the reader to [9] for more details.

Lemma 2.1. Given two Banach spaces $\mathcal{X}$ and $\mathcal{Y}$, assume that the bilinear forms $B_{1}: \mathcal{X} \times \mathcal{X} \rightarrow \mathcal{X}$ and $B_{2}: \mathcal{X} \times \mathcal{Y} \rightarrow \mathcal{Y}$ satisfy the estimates $\left\|B_{1}(u, v)\right\|_{\mathcal{X}} \leq$ $\alpha_{1}\|u\|_{\mathcal{X}}\|v\|_{\mathcal{X}}$ and $\left\|B_{2}(v, T)\right\|_{\mathcal{Y}} \leq \alpha_{2}\|v\|_{\mathcal{X}}\|T\|_{\mathcal{Y}}$ with positive constants $\alpha_{1}$ and $\alpha_{2}$, for all $u, v \in \mathcal{X}$ and $T \in \mathcal{Y}$. Let the linear operator $L: \mathcal{Y} \rightarrow \mathcal{Y}$ be continuous and satisfy the inequality $\|L T\|_{\mathcal{Y}} \leq \eta\|T\|_{\mathcal{Y}}$ with a constant $\eta<1$. For every $\left(y_{1}, y_{2}\right) \in \mathcal{X} \times \mathcal{Y}$ such that $\left\|y_{1}\right\|_{\mathcal{X}}+\left\|y_{2}\right\|_{\mathcal{Y}} \leq \alpha_{1}(1-\eta)^{2} /\left[2 \alpha_{1}+\alpha_{2}\right]^{2}$, there exists a solution $(v, T) \in \mathcal{X} \times \mathcal{Y}$ to system (2.1). This is the unique solution satisfying the condition $\left(\|v\|_{\mathcal{X}}+\|T\|_{\mathcal{Y}}\right)<(1-\eta) /\left(2 \alpha_{1}+\alpha_{2}\right)$.

Proof. Let us choose arbitrary $R \in\left((1-\eta)\left[2\left(\alpha_{1}+\alpha_{2}\right)\right]^{-1},(1-\eta)\left[2 \alpha_{1}+\alpha_{2}\right]^{-1}\right)$. It suffices to show that the nonlinear operator $\Theta(v, T) \equiv\left(y_{1}+B_{1}(v, v)+L T, y_{2}+\right.$ $\left.B_{2}(v, T)\right)$ is the contraction on the closed ball $B(0, R)=\{(v, T) \in \mathcal{X} \times \mathcal{Y}$ : $\left.\|v\|_{\mathcal{X}}+\|T\|_{\mathcal{Y}} \leq R\right\}$. Here, we supplement the product space $\mathcal{X} \times \mathcal{Y}$ with the norm $\|(v, t)\| \equiv\|v\|_{\mathcal{X}}+\|T\|_{\mathcal{Y}}$. First note that for every $(v, T) \in B(0, R)$ we have

$$
\|\Theta(v, T)\| \leq\left\|y_{1}\right\|_{\mathcal{X}}+\alpha_{1} R^{2}+\eta R+\left\|y_{2}\right\|_{\mathcal{Y}}+\alpha_{2} R^{2} .
$$

Now, observe that, by the smallness assumption on $y_{1}$ and $y_{2}$, we obtain $\left\|y_{1}\right\|_{\mathcal{X}}+$ $\left\|y_{1}\right\|_{\mathcal{Y}} \leq(1-\eta) R-\left(\alpha_{1}+\alpha_{2}\right) R^{2}$. Indeed, the infimum of the right-hand side with respect to $R \in\left((1-\eta)\left[2\left(\alpha_{1}+\alpha_{2}\right)\right]^{-1},(1-\eta)\left[2 \alpha_{1}+\alpha_{2}\right]^{-1}\right)$ equals $\alpha_{1}(1-\eta)^{2} /\left[2 \alpha_{1}+\right.$ $\left.\alpha_{2}\right]^{2}$. Hence, $\|\Theta(v, T)\| \leq R$. To prove that $\Theta$ is the contraction in $B(0, R)$, we proceed as follows. For any $(v, T),(\widetilde{v}, \widetilde{T}) \in B(0, R)$, we estimate

$$
\begin{aligned}
\|\Theta(v, T)-\Theta(\widetilde{v}, \widetilde{T})\| \leq & 2 \alpha_{1} R\|v-\widetilde{v}\|_{\mathcal{X}}+\eta\|T-\widetilde{T}\|_{\mathcal{Y}} \\
& +\alpha_{2} R\left(\|v-\widetilde{v}\|_{\mathcal{X}}+\|T-\widetilde{T}\|_{\mathcal{Y}}\right) \\
\leq & \left(R\left(2 \alpha_{1}+\alpha_{2}\right)+\eta\right)\|(v, T)-(\widetilde{v}, \widetilde{T})\| .
\end{aligned}
$$

The proof is complete because $R\left(2 \alpha_{1}+\alpha_{2}\right)+\eta<1$.

Due to Lemma 2.1, we should estimate each term appearing on the right-hand side of equations (1.4) and (1.5).

Lemma 2.2. For every $u_{0} \in \mathcal{P M}^{2}, T_{0} \in \mathcal{P} \mathcal{M}^{0}$, and $F \in \mathcal{C}_{w}\left(\left[0, \infty\left[, \mathcal{P M}^{0}\right)\right.\right.$ we have $S(t) v_{0} \in \mathcal{X}$ and $\int_{0}^{t} S(t-\tau) \mathbb{P} F(\tau) d \tau \in \mathcal{X}$ with the estimates

$$
\left\|S(t) v_{0}\right\|_{\mathcal{P M}^{2}} \leq\left\|v_{0}\right\|_{\mathcal{P M}^{2}} \quad \text { and } \quad\left\|\int_{0}^{t} S(t-\tau) \mathbb{P} F(\tau) d \tau\right\|_{\mathcal{P M}^{2}} \leq \sup _{t>0}\|F(t)\|_{\mathcal{P M}^{0}}
$$

Moreover, for any $a \geq 0$, it follows that $S(\cdot) T_{0} \in \mathcal{Y}_{a}$ and the following estimates hold true:

$$
\left\|S(t) T_{0}\right\|_{\mathcal{P M}_{0}} \leq\left\|T_{0}\right\|_{\mathcal{P M}_{0}} \quad \text { and } \quad t^{a / 2}\left\|S(t) T_{0}\right\|_{\mathcal{P M}^{a}} \leq C\left\|T_{0}\right\|_{\mathcal{P M}^{0}} .
$$


Proof. For the proofs of (2.2), the reader is referred to [4, Lemmata 4.2 and 4.3]. The reasoning in the case of the first inequality in (2.3) is completely analogous; hence, we skip it. By the definition of the norm in $\mathcal{P M}^{a}$, we get

$$
t^{a / 2}\left\|S(t) T_{0}\right\|_{\mathcal{P M}^{a}} \leq\left\|T_{0}\right\|_{\mathcal{P M}^{0}} \sup _{\xi \in \mathbb{R}^{3}}\left(t|\xi|^{2}\right)^{a / 2} e^{-t|\xi|^{2}}=C\left\|T_{0}\right\|_{\mathcal{P M}^{0}}
$$

which completes the proof that $S(\cdot) T_{0} \in \mathcal{Y}_{a}$.

The estimates of $B_{1}, B_{2}$ and $L$ required by Lemma 2.1 are shown in the following three lemmata. The bilinear form $B_{1}$ is studied in the first of them.

Lemma 2.3. There exists a constant $\alpha_{1}>0$ such that for every $u, v \in \mathcal{X}$, we have

$$
\left\|\int_{0}^{t} S(t-\tau) \mathbb{P} \nabla \cdot(u \otimes v)(\tau) d \tau\right\|_{\mathcal{P M}^{2}} \leq \alpha_{1}\left(\sup _{t>0}\|u(t)\|_{\mathcal{P M}^{2}}\right)\left(\sup _{t>0}\|v(t)\|_{\mathcal{P M}^{2}}\right)
$$

for all $t>0$

This is the well-known estimate which appeared in the study of the Navier-Stokes system. Hence, the reader is referred to [4, Prop. 4.1] for the proof of Lemma 2.3.

Now, we deal with the bilinear form $B_{2}$.

Lemma 2.4. Assume that $1<a<2$. There exists a constant $\alpha_{2}>0$ such that for every $v \in \mathcal{X}$ and $T \in \mathcal{Y}_{a}$ the following estimate holds true:

$$
\left\|\int_{0}^{t} \nabla S(t-\tau)(v T)(\tau) d \tau\right\|_{\mathcal{Y}_{a}} \leq \alpha_{2}\|v\|_{\mathcal{X}}\|T\|_{\mathcal{Y}_{a}}
$$

for all $t>0$.

Proof. This result is deduced from the equality $\left.|\cdot|\right|^{-2} *|\cdot|^{-a}=C|\cdot|^{-a+1}$ which holds true for every $a \in(1,3)$ in the space $\mathbb{R}^{3}$. Indeed, using the definition of the $\mathcal{Y}_{a}$-norm, we have

$$
\begin{aligned}
& \left|\int_{0}^{t} i \xi e^{-(t-\tau)|\xi|^{2}}(\widehat{v} * \widehat{T})(\tau, \xi) d \tau\right| \\
& \quad \leq\left(\int_{0}^{t}|\xi| e^{-(t-\tau)|\xi|^{2}}|\xi|^{-2} *|\xi|^{-a} \tau^{-a / 2} d \tau\right)\|v\|_{\mathcal{X}}\|T\|_{\mathcal{Y}_{a}} \\
& \quad=C\|v\|_{\mathcal{X}}\|T\|_{\mathcal{Y}_{a}} \int_{0}^{t}|\xi|^{2-a} e^{-(t-\tau)|\xi|^{2}} \tau^{-a / 2} d \tau .
\end{aligned}
$$

Hence, by the equality

$$
\sup _{\xi \in \mathbb{R}^{3}}|\xi|^{b} e^{-(t-\tau)|\xi|^{2}}=C(t-\tau)^{-b / 2}
$$

with $b=2-a$, we may estimate the right-hand side of (2.5) by $C\|v\|_{\mathcal{X}}\|T\|_{\mathcal{Y}_{a}}$ because the integral $\int_{0}^{t}(t-\tau)^{-1+a / 2} \tau^{-a / 2} d \tau$ is finite and independent of $t$ for any $a \in(0,2)$. Consequently, we obtain the estimate of the first component of the $\mathcal{Y}_{a}$-norm

$$
\sup _{t>0}\left\|\int_{0}^{t} \nabla S(t-\tau)(v T)(\tau) d \tau\right\|_{\mathcal{P M}^{0}} \leq C_{1}\|v\|_{\mathcal{X}}\|T\|_{\mathcal{Y}_{a}}
$$


Now, let us deal with the second term of the $\mathcal{Y}_{a}$-norm. Note first the following two elementary inequalities:

$$
|\xi|^{2-a} \int_{t / 2}^{t} e^{-(t-\tau)|\xi|^{2}} \tau^{-a / 2} d \tau \leq(t / 2)^{-a / 2}|\xi|^{2-a} \int_{t / 2}^{t} e^{-(t-\tau)|\xi|^{2}} d \tau \leq C t^{-a / 2}|\xi|^{-a}
$$

and (by (2.6) with $t-\tau$ replaced by $t / 2$ and with $b=2$ )

$$
|\xi|^{2-a} \int_{0}^{t / 2} e^{-(t-\tau)|\xi|^{2}} \tau^{-a / 2} d \tau \leq|\xi|^{2-a} e^{-(t / 2)|\xi|^{2}} \int_{0}^{t / 2} \tau^{-a / 2} d \tau=C|\xi|^{-a} t^{-a / 2}
$$

Hence, proceeding as in (2.5), we obtain

$$
\begin{aligned}
& \left|\int_{0}^{t} i \xi e^{-(t-\tau)|\xi|^{2}}(\widehat{v} * \widehat{T})(\tau, \xi) d \tau\right| \\
& \quad \leq C\|v\|_{\mathcal{X}}\|T\|_{\mathcal{Y}_{a}}|\xi|^{2-a}\left(\int_{0}^{t / 2}+\int_{t / 2}^{t}\right) e^{-(t-\tau)|\xi|^{2}} \tau^{-a / 2} d \tau \\
& \quad \leq C|\xi|^{-a} t^{-a / 2}\|v\|_{\mathcal{X}}\|T\|_{\mathcal{Y}_{a}}
\end{aligned}
$$

which immediately leads to the inequality

$$
\sup _{t>0} t^{a / 2}\left\|\int_{0}^{t} \nabla S(t-\tau)(v T)(\tau) d \tau\right\|_{\mathcal{P M}^{a}} \leq C_{2}\|v\|_{\mathcal{X}}\|T\|_{\mathcal{Y}_{a}} .
$$

The proof of Lemma 2.4 is complete by (2.7) and (2.8) with $\alpha_{2}=C_{1}+C_{2}$.

Finally, we deal with the linear operator $L$.

Lemma 2.5. Let $1<a<2$. For every $T \in \mathcal{Y}_{a}$, the following estimate holds true:

for all $t>0$.

$$
\left\|\int_{0}^{t} S(t-\tau) \mathbb{P} T(\tau) d \tau\right\|_{\mathcal{P M}^{2}} \leq \sup _{t>0}\|T(t)\|_{\mathcal{P M}^{0}}
$$

Note that the estimate from Lemma 2.5 is, in fact, presented as the second inequality in (2.2) with $F$ replaced by $T$; hence here we skip the proof.

Proof of Theorem 1.1. Lemmata 2.3, 2.4, and 2.5 provide the estimates required by Lemma 2.1. Hence Theorem 1.1 is proved under the assumption $\eta=|\beta|<1$.

Proof of Corollary 1.3. Let $(v, T)$ be the solution of (1.1) corresponding to the initial datum $\left(v_{0}, T_{0}\right)$ and the external force $F$ constructed via Theorem 1.1. By the scaling invariance of system (1.1) we get that the functions given by (1.2) are also solutions with the same initial condition and the external force. Hence, by the uniqueness result of Theorem 1.1, the solution $(v, T)$ of $(1.1)$ is self-similar because the norms in $\mathcal{X}$ and $\mathcal{Y}_{a}$ are invariant under the rescaling (1.2).

\section{LARGE TIME BEHAVIOR OF SOLUTIONS}

We begin by preliminary estimates.

Lemma 3.1. There exists a constant $C>0$ such that for all $t>0,0<\tau<t$, $v \in \mathcal{P} \mathcal{M}^{2}$ and $T \in \mathcal{Y}_{a}$, the following estimates hold true:

$$
\begin{gathered}
\|S(t-\tau) \mathbb{P} T(\tau)\|_{\mathcal{P M}^{2}} \leq C(t-\tau)^{-1}\|T\|_{\mathcal{Y}_{a}}, \\
\|\nabla S(t-\tau)(v T)(\tau)\|_{\mathcal{P M}^{0}} \leq C(t-\tau)^{-1+a / 2} \tau^{-a / 2}\|v\|_{\mathcal{X}}\|T\|_{\mathcal{Y}_{a}},
\end{gathered}
$$


and

$$
\|\nabla S(t-\tau)(v T)(\tau)\|_{\mathcal{P M}^{a}} \leq C(t-\tau)^{-1} \tau^{-a / 2}\|v\|_{\mathcal{X}}\|T\|_{\mathcal{Y}_{a}} .
$$

Proof. By the definition of the $\mathcal{P} \mathcal{M}^{2}$-norm and (2.6) with $b=2$, we immediately obtain

$$
\|S(t-\tau) T(\tau)\|_{\mathcal{P M}^{2}} \leq\|T(\tau)\|_{\mathcal{P M}^{0}} \sup _{\xi \in \mathbb{R}^{3}}|\xi|^{2} e^{-(t-\tau)|\xi|^{2}} \leq C(t-\tau)^{-1}\|T\|_{\mathcal{Y}_{a}} .
$$

Next, we deal with the $\mathcal{P} \mathcal{M}^{0}$-norm. By the calculations as in the proof of Lemma 2.4 (cf. (2.7)) we have

$$
\begin{aligned}
\left|i \xi e^{-(t-\tau)|\xi|^{2}} \widehat{v} * \widehat{T}(\xi)\right| & \leq C|\xi|^{2-a} e^{-(t-\tau)|\xi|^{2}}\|v(\tau)\|_{\mathcal{P M}^{2}}\|T(\tau)\|_{\mathcal{P M}^{a}} \\
& \leq C(t-\tau)^{-1+a / 2} \tau^{-a / 2}\|v(\tau)\|_{\mathcal{P M}^{2}}\left(\tau^{a / 2}\|T(\tau)\|_{\mathcal{P M}^{a}}\right) .
\end{aligned}
$$

Next, we study the $\mathcal{P M}^{a}$-norm

$$
\begin{aligned}
|\xi|^{a}\left|i \xi e^{-(t-\tau)|\xi|^{2}} \widehat{v} * \widehat{T}(\xi)\right| & \leq|\xi|^{2} e^{-(t-\tau)|\xi|^{2}}\|v(\tau)\|_{\mathcal{P M}^{2}}\|T(\tau)\|_{\mathcal{P M}^{a}} \\
& \leq C(t-\tau)^{-1} \tau^{-a / 2}\|v(\tau)\|_{\mathcal{P M}^{2}}\left(\tau^{a / 2}\|T(\tau)\|_{\mathcal{P M}^{a}}\right)
\end{aligned}
$$

by (2.6) with $b=2$. The proof of Lemma 3.1 is complete.

Now we recall how to deal with the external force. The following lemma was shown in [4, Lemma 5.1]; hence we skip its proof.

Lemma 3.2. Assume that $F \in \mathcal{C}_{w}\left(\left[0, \infty\left[, \mathcal{P M}^{0}\right)\right.\right.$ satisfies $\lim _{t \rightarrow \infty}\|F(t)\|_{\mathcal{P M}^{0}}=0$. Then,

$$
\lim _{t \rightarrow \infty}\left\|\int_{0}^{t} S(t-\tau) F(\tau) d \tau\right\|_{\mathcal{P M}^{2}}=0 .
$$

We are in a position to show our main result on the large time behavior of solutions to system (1.1).

Proof of Theorem 1.5. Let $(v, T)$ and $(\bar{v}, \bar{T})$ be two solutions of (1.1) constructed in Theorem 1.1. Recall that, by Lemma 2.1, one may choose $R_{0}<(1-\eta) /\left(2 \alpha_{1}+\alpha_{2}\right)$ with the constants $\alpha_{1}, \alpha_{2}$ and $\eta=|\beta|$ obtained in Lemmata 2.3, 2.4, and 2.5, respectively, such that

$$
\|v\|_{\mathcal{X}}+\|T\|_{\mathcal{Y}_{a}} \leq R_{0} \quad \text { and } \quad\|\bar{v}\|_{\mathcal{X}}+\|\bar{T}\|_{\mathcal{Y}_{a}} \leq R_{0}
$$

We subtract the integral equation (1.4) for $\bar{v}$ and $\bar{T}$ from the analogous expression for $v$ and $T$ to get

$$
\begin{aligned}
v(t)-\bar{v}(t) & =S(t)\left(v_{0}-\bar{v}_{0}\right)+\int_{0}^{t} \nabla S(t-\tau) \mathbb{P}((v \otimes v)(\tau)-(\bar{v} \otimes \bar{v})(\tau)) d \tau \\
& +\beta \int_{0}^{t} S(t-\tau) \mathbb{P}(T(\tau)-\bar{T}(\tau)) d \tau+\int_{0}^{t} S(t-\tau) \mathbb{P}(F(\tau)-\bar{F}(\tau)) d \tau
\end{aligned}
$$

We proceed in a same way with equation (1.5):

$$
T(t)-\bar{T}(t)=S(t)\left(T_{0}-\bar{T}_{0}\right)+\int_{0}^{t} \nabla S(t-\tau)((v T)(\tau)-(\bar{v} \bar{T})(\tau)) d \tau .
$$


Now, to shorten the notation, we introduce the auxiliary function

$$
\begin{aligned}
h(t) \equiv & \left\|S(t)\left(v_{0}-\bar{v}_{0}\right)\right\|_{\mathcal{P M}^{2}}+\left\|\int_{0}^{t} S(t-\tau) \mathbb{P}(F(\tau)-\bar{F}(\tau)) d \tau\right\|_{\mathcal{P M}^{2}} \\
& +\left\|S(t)\left(T_{0}-\bar{T}_{0}\right)\right\|_{\mathcal{P M}^{0}}+t^{a / 2}\left\|S(t)\left(T_{0}-\bar{T}_{0}\right)\right\|_{\mathcal{P M}^{a}} .
\end{aligned}
$$

It follows immediately from Lemma 2.2 that $h \in L^{\infty}(0, \infty)$. Moreover, by assumption (1.7) (note the inequality $t^{a / 2}\left\|S(t) T_{0}\right\|_{\mathcal{P M}^{a}} \leq C\left\|S(t / 2) T_{0}\right\|_{\mathcal{P M}^{0}}$ being the immediate consequence of (2.4)) and Lemma 3.2, we have $\lim _{t \rightarrow \infty} h(t)=0$.

By computing the $\mathcal{P} \mathcal{M}^{2}$-norm of (3.5) and the $\mathcal{P \mathcal { M } ^ { 0 }}$-norm as well as $\mathcal{P} \mathcal{M}^{a}$-norms multiplied by $t^{a / 2}$ of (3.6) and next adding the resulting inequalities, we obtain

$$
\begin{aligned}
& \|v(t)-\bar{v}(t)\|_{\mathcal{P M}^{2}}+\|T(t)-\bar{T}(t)\|_{\mathcal{P M}^{0}}+t^{a / 2}\|T(t)-\bar{T}(t)\|_{\mathcal{P M}^{a}} \\
& \leq \quad h(t)+\left\|\int_{0}^{t} \nabla S(t-\tau) \mathbb{P}[(v \otimes v)(\tau)-(\bar{v} \otimes \bar{v})(\tau)] d \tau\right\|_{\mathcal{P M}^{2}} \\
& +|\beta|\left\|\int_{0}^{t} S(t-\tau) \mathbb{P}[T(\tau)-\bar{T}(\tau)] d \tau\right\|_{\mathcal{P M}^{2}} \\
& +\left\|\int_{0}^{t} S(t-\tau)[(v T)(\tau)-(\bar{v} \bar{T})(\tau)] d \tau\right\|_{\mathcal{P M}^{0}} \\
& +t^{a / 2}\left\|\int_{0}^{t} S(t-\tau)[(v T)(\tau)-(\bar{v} \bar{T})(\tau)] d \tau\right\|_{\mathcal{P M}^{a}} .
\end{aligned}
$$

Given $\delta \in(0,1)$, we decompose the second term on the right-hand side of (3.7) as $\int_{0}^{\delta t} \ldots d \tau+\int_{\delta t}^{t} \ldots d \tau$ and we estimate each term separately. We use [4, estimate $(5.5)]$ in the case of the integral over $[0, \delta t]$ in order to show that

$$
\begin{aligned}
& \int_{0}^{\delta t}\|\nabla S(t-\tau) \mathbb{P}[(v \otimes v)(\tau)-(\bar{v} \otimes \bar{v})(\tau)]\|_{\mathcal{P M}^{2}} d \tau \\
& \leq C \int_{0}^{\delta t}(t-\tau)^{-1}\|v(\tau)-\bar{v}(\tau)\|_{\mathcal{P M}^{2}} d \tau\left(\sup _{\tau>0}\|v(\tau)\|_{\mathcal{P M}^{2}}+\sup _{\tau>0}\|\bar{v}(\tau)\|_{\mathcal{P M}^{2}}\right) \\
& \leq 4 C R_{0}^{2} \log \left(\frac{1}{1-\delta}\right) .
\end{aligned}
$$

The second term containing the integral term $\int_{\delta t}^{t} \cdots d \tau$ is treated directly by Lemma 2.3 (see also [5, estimate (5.6)]) to obtain

$$
\begin{aligned}
& \| \int_{\delta t}^{t} \nabla S(t-\tau) \mathbb{P}[(v \otimes v)(\tau)-(\bar{v} \otimes \bar{v})(\tau)] d \tau \|_{\mathcal{P M}^{2}} \\
& \leq 2 \alpha_{1} R_{0} \sup _{\delta t \leq \tau \leq t}\|v(\tau)-\bar{v}(\tau)\|_{\mathcal{P M}^{2}}
\end{aligned}
$$

We proceed in the analogous way with the third term on the right-hand side of (3.7) using (3.1) and Lemma 2.5 to bound it as follows:

$$
\begin{aligned}
|\beta|\left\|\int_{0}^{t} S(t-\tau) \mathbb{P}[T(\tau)-\bar{T}(\tau)] d \tau\right\|_{\mathcal{P M}^{2}} \\
\leq|\beta|\left(C R_{0} \log \left(\frac{1}{1-\delta}\right)+\sup _{\delta t \leq \tau \leq t}\|T(\tau)-\bar{T}(\tau)\|_{\mathcal{P} \mathcal{M}^{0}}\right) .
\end{aligned}
$$


In a similar way, using estimates (3.2)-(3.3) for the integrals on $[0, \delta t]$ and Lemma 2.4 for $\tau \in[\delta t, t]$, we obtain the following inequalities for the forth and fifth terms on the right-hand side of (3.7):

$$
\begin{aligned}
\| \int_{0}^{t} S(t- & \tau)[(v T)(\tau)-(\bar{v} \bar{T})(\tau)] d \tau \|_{\mathcal{P M}^{0}} \\
& +t^{a / 2}\left\|\int_{0}^{t} S(t-\tau)[(v T)(\tau)-(\bar{v} \bar{T})(\tau)] d \tau\right\|_{\mathcal{P M}^{a}} \\
\leq & C R_{0}^{2}\left(\int_{0}^{\delta}(1-s)^{-1+a / 2} s^{-a / 2} d s+\int_{0}^{\delta}(1-s)^{-1} s^{-a / 2} d s\right) \\
& +\alpha_{2} R_{0}\left(\sup _{\delta t \leq \tau \leq t}\|v(\tau)-\bar{v}(\tau)\|_{\mathcal{P M}^{2}}+\sup _{\delta t \leq \tau \leq t} \tau^{a / 2}\|T(\tau)-\bar{T}(\tau)\|_{\mathcal{P M}^{a}}\right) .
\end{aligned}
$$

Next, following [4], we put

$$
A=\limsup _{t \rightarrow \infty}\left(\|v(t)-\bar{v}(t)\|_{\mathcal{P M}^{2}}+\|T(t)-\bar{T}(t)\|_{\mathcal{P M}^{0}}+t^{a / 2}\|T(t)-\bar{T}(t)\|_{\mathcal{P M}^{a}}\right),
$$

where "lim $\sup _{t \rightarrow \infty}$ " is understood as " $\lim _{k \in \mathbb{N}, k \rightarrow \infty} \sup _{t \geq k}$ ". This number is finite due to the properties of the solutions $(v, T)$ and $(\bar{v}, \bar{T})$ from Theorem 1.1, and our aim is to prove that $A=0$. For this reason we compute "lim $\sup _{t \rightarrow \infty}$ " of both sides of inequality (3.7). Obviously, we have

$$
\begin{gathered}
\limsup _{t \rightarrow \infty}\left(\sup _{\delta t \leq \tau \leq t}\|v(\tau)-\bar{v}(\tau)\|_{\mathcal{P M}^{2}}+\sup _{\delta t \leq \tau \leq t}\|T(\tau)-\bar{T}(\tau)\|_{\mathcal{P M}^{0}}\right. \\
\left.\quad+\sup _{\delta t \leq \tau \leq t}(\tau)^{a / 2}\|T(\tau)-\bar{T}(\tau)\|_{\mathcal{P M}^{a}}\right) \leq A .
\end{gathered}
$$

Recalling that $\lim _{t \rightarrow \infty} h(t)=0$, we obtain from (3.7)-(3.13) the inequality

$$
A \leq g(\delta)+\left(2 \alpha_{1} R_{0}+\alpha_{2} R_{0}+\eta\right) A
$$

with

$$
g(\delta)=C R_{0}^{2}\left(\log \left(\frac{1}{1-\delta}\right)+\int_{0}^{\delta}(1-s)^{-1+a / 2} s^{-a / 2} d s+\int_{0}^{\delta}(1-s)^{-1} s^{-a / 2} d s\right)
$$

which tends to 0 as $\delta \searrow 0$. Since $\delta \in(0,1)$ can be arbitrarily small and $2 \alpha_{1} R_{0}+$ $\alpha_{2} R_{0}+\eta<1$ by our assumption on $R_{0}$, we obtain $A=0$ and the proof of Theorem 1.5 is complete.

\section{REFERENCES}

[1] Bejan, A; Convection Heat Transfer, 2nd edition, John Wiley and Sons Inc., 1995.

[2] Cannon, J. R. \& DiBenedetto, E., The initial value problem for the Boussinesq equations with data in $L^{p}$. Approximation methods for Navier-Stokes problems (Proc. Sympos., Univ. Paderborn, Paderborn, 1979), 129-144, Lecture Notes in Math., 771, Springer, Berlin, 1980. MR565993 (81f:35101)

[3] Cannone, M., Harmonic analysis tools for solving the incompressible Navier-Stokes equations, Handbook of mathematical fluid dynamics. Vol. III, 161-244, North-Holland, Amsterdam, 2004. MR2099035 (2006c:35216)

[4] Cannone, M. \& Karch, G., Smooth or singular solutions to the Navier-Stokes system?, J. Differential Equations, 197 (2004), 247 - 274. MR2034160 (2005g:35228) 
[5] Cannone, M. \& Karch, G., About regularized Navier-Stokes equations, J. Math. Fluid Mech. 7 (2005), 1-28. MR2127740 (2005m:35219)

[6] Hishida, T., On a class of stable steady flows to the exterior convection problem, J. Differential Equations 141 (1997), 54-85. MR1485942 (98k:35159)

[7] Karch, G., Scaling in nolinear parabolic equations, J. Math. Anal. Appl., 234 (1999), 534 558. MR1689406 (2000h:35068)

[8] Landau, L. D. \& Lifchitz, E. M., Theorical Physics: Fluid Mechanics, 2nd edition, Pergamon Press, 1987. MR961259 (89i:00006)

[9] Prioux, N., Asymptotic stability results for some nonlinear evolution equations, Adv. Math. Sci. Appl., 17 (2007), 37-65.

Instytut Mathematyczny, UniWersytet Wroclawski, Pl. GrunWaldzki 2/4, 50-384, Wroclaw, Poland

E-mail address: karch@math.uni.wroc.pl

Laboratoire D'Analyse et de Mathématiques Appliquées, Université de Marne-laVallée, Cité Descartes-5, Bd Descartes, Champs-sur-Marne, 77454 Marne-la-Vallée Cedex 2, France

E-mail address: nicolas.prioux@univ-mlv.fr 